Artigo Original

Original Article

Descritores

Voz

Dor

Treinamento de voz

Percepção da dor

Auto-avaliação

Keywords

Voice

Pain

Voice training

Pain perception

Self-assessment
Endereço para correspondência:

Thays Vaiano

R. Doutor Tirso Martins, 44/23,

Vila Mariana, São Paulo (SP), Brasil,

CEP: 04035-000.

E-mail: tvaiano@uol.com.br

\section{Dores corporais em coristas eruditos}

\section{Body pain in classical choral singers}

\section{RESUMO}

Objetivo: Identificar e caracterizar dores corporais em coristas eruditos. Métodos: Foi aplicado um questionário autoexplicativo a 50 coristas eruditos e 150 sujeitos da população geral, com questões de caracterização pessoal e sobre a presença e frequência de 13 tipos de dores corporais: cabeça, pescoço, nuca, ombros, costas, peito, mão, ouvido, língua, articulação temporomandibular, garganta e dor ao falar. Resultados: Coristas eruditos relataram menos dores do que a população geral. Os tipos de dores mais relatados por coristas foram garganta, costas e ombros, respectivamente. Conclusão: A baixa ocorrência de dores corporais em coristas eruditos sugere que o uso vocal saudável, aliado ao treino da musculatura respiratória e de produção vocal, pode oferecer uma maior resistência musculoesquelética, prevenindo o aparecimento de doenças associadas a ela.

\begin{abstract}
Purpose: To identify and characterize the presence of body pain related to voice usage in choral singers. Methods: A questionnaire investigating the occurrence of voice problems, vocal self-evaluation, and a report of body aches was given to 50 classical choral singers and 150 participants who were non-singers. Thirteen types of aches were investigated that were distributed into two groups: larynx proximal ache (temporomandibular joint, tongue, sore throat, neck, back of the neck, shoulder, and pain while speaking) and distal ache (headache, backache, chest, arms, hands, and ear ache). Results: Classical choral singers had less presence of pain than the general population. The most related pain types reported in singers were sore throat, chest, and shoulder, respectively. Conclusion: Reduced vocal signs of pain in singers may suggest that singers can benefit from vocal training once they have better voice usage due to voice practice, offering a protective effect to the development of voice disorders since voice training builds up a better musculoskeletal endurance.
\end{abstract}

Trabalho realizado no Centro de Estudos da Voz - CEV - São Paulo (SP), Brasil.

(1) Centro de Estudos da Voz - CEV - São Paulo (SP), Brasil.

(2) Ativox - São Paulo (SP), Brasil.

(3) Universidade Federal de São Paulo - UNIFESP - São Paulo (SP), Brasil.

Conflito de interesses: nada a declarar. 


\section{INTRODUÇÃO}

A dor é definida pela International Association for the Study of Pain (IASP) como uma "experiência sensitiva e emocional desagradável associada ou relacionada à lesão real ou potencial dos tecidos". Segundo a instituição, a dor musculoesquelética é a mais comum. Por isso, em uma de suas últimas campanhas, a IASP chamou atenção para a dor incapacitante vivenciada por pessoas de todo o mundo que sofrem de lesões musculoesqueléticas ${ }^{(1)}$. A associação entre dores corporais e sintomas vocais tem sido observada clinicamente, mas ainda não é adequadamente investigada. Quando um indivíduo usa a voz de forma inadequada, com tensão e esforço para falar, pode sentir desconforto ou mesmo dor à fonação. Esta dor, chamada de odinofonia, é considerada um sinal e sintoma de problema vocal ${ }^{(2)}$.

A presença de um problema vocal pode se manifestar por diversos sintomas e pode ser causado por alterações em estruturas e sistemas que não estejam necessariamente ligados à laringe ${ }^{(3,4)}$. Uma pesquisa investigou os padrões músculoesqueléticos em pacientes com distúrbios vocais e encontrou queixas musculares em $69 \%$ dos sujeitos ${ }^{(5)}$. Além disso, a fraqueza ou o desequilíbrio de alguns grupos musculares pode agravar os sintomas vocais por reduzir a estrutura de apoio necessária para a manutenção da função de forma ideal ${ }^{(2)}$.

A disfonia por tensão muscular é provavelmente a causa mais comum de disfonia funcional e contempla uma série de queixas por tensão ou desconforto muscular como dor à fonação, sensação de tensão física, dor na garganta, na cabeça, dor ou desconforto na articulação temporomandibular (ATM), pescoço e peito ${ }^{(6)}$. O treino vocal pode ser considerado uma medida profilática em relação aos distúrbios vocais, uma vez que estimula uso equilibrado da musculatura envolvida na produção da voz e na fala.

Profissionais da voz tendem a dar maior importância e atenção aos aspectos que envolvam a saúde vocal, pois qualquer variação na voz pode comprometer sua performance. Os cantores eruditos têm uma importante demanda vocal e dependem de uma voz limpa e equilibrada para que tenham um bom desempenho profissional. Estudantes de conservatórios parecem dar mais importância à saúde vocal e estão mais atentos aos cuidados necessários para não desenvolver um problema de voz do que a população geral ${ }^{(7)}$. Para investigar tal possibilidade, para esta pesquisa, foram selecionados cantores de coral erudito, visto que este estilo de canto exige técnica e uma boa autopercepção quanto ao uso da voz. Acreditamos que o treinamento vocal proporcione maior resistência musculoesquelética e, por isso, reduza a ocorrência de dores corporais, principalmente na região próxima à laringe.

O objetivo deste estudo é identificar a presença de dores corporais em coristas eruditos, verificar sua correlação com ocorrência de problemas vocais, absenteísmo e procura por tratamentos específicos e comparar com os resultados obtidos pela população geral.

\section{MÉTODOS}

A presente pesquisa foi aprovada pelo Comitê de Ética do Centro de Estudos da Voz (CEV), parecer número 1050/11. Trata-se de um estudo descritivo transversal. Os participantes responderam um questionário, autoexplicativo, que investigava informações sobre voz, trabalho e presença de dores corporais ${ }^{(8)}$. A coleta dos dados foi realizada nos meses de outubro de 2007 a janeiro de 2008, na cidade de São Paulo. Todos os participantes assinaram o Termo de Consentimento Livre e Esclarecido.

Participaram 50 membros de um coral erudito profissional ( 29 mulheres e 21 homens) com idades entre 18 e 57 anos e 150 sujeitos da população geral ( 87 mulheres e 63 homens) com idades de 18 a 63 anos, que não exerciam atividade de canto erudito ou popular profissionalmente. Todos os participantes do coral exerciam outra profissão e a participação do coral, apesar de profissional, não é remunerada. O número de sujeitos da população geral foi o maior possível que permitisse comparação estatística com o grupo dos cantores eruditos.

Todos os sujeitos responderam um questionário autoexplicativo (Anexos 1 e 2) composto por 11 questões fechadas que foram divididas em cinco categorias de informação: 1. Dados de identificação (gênero, profissão e tempo de atuação); 2. Fatores relacionados ao exercício profissional (profissão, tempo de atuação e horas diárias de trabalho); 3. Autoavaliação da voz; 4. Dados referentes à voz (relato de ocorrência de problemas vocais durante o exercício profissional, necessidade de se afastar do trabalho devido a problemas vocais e procura por especialista médico otorrinolaringologista e/ou fonoaudiólogo devido a problemas vocais); 5 . Localização de dores em diferentes regiões do corpo durante e/ou após o uso profissional da voz.

Os dados de identificação e fatores relacionados ao exercício profissional foram usados para caracterizar a amostra. Para os cantores eruditos as perguntas foram direcionadas a questões específicas da prática de canto (Anexo 1) e, para população geral as perguntas foram genéricas e, portanto, aplicáveis a sujeitos de qualquer profissão (Anexo 2). Os dados de identificação e fatores relacionados ao exercício profissional, por serem perguntas diferentes entre os grupos, não foram utilizados nas análises estatísticas.

Na investigação da autoavaliação vocal a voz deveria ser classificada pelo próprio sujeito por meio da escala de Likert, de cinco pontos que continha as seguintes opções de resposta: ótima, boa, razoável, ruim ou péssima. As questões relacionadas à voz e à atividade de trabalho tiveram como objetivo verificar se o sujeito já teve problemas vocais durante a atuação profissional, se já precisou se afastar do trabalho devido a problemas de voz, se já consultou médico otorrinolaringologista devido a problemas de voz e se já consultou fonoaudiólogo devido a problemas de voz.

A presença de dores corporais foi investigada em relação à ocorrência e frequência. Foram pesquisados 13 tipos de dores corporais: dor de cabeça, dor na língua, dor na ATM, dor na garganta, dor na nuca, dor nos ombros, dor nas costas/coluna, dor no pescoço, dor no peito, dor nos braços, dor nas mãos, dor nos ouvidos e dor ao falar. Para determinar a frequência, os 
participantes poderiam responder, por meio de uma escala de Likert de cinco pontos: nunca, às vezes, muitas vezes, quase sempre e sempre.

Para análise dos resultados, a localização das dores foi agrupada em duas categorias: dores proximais e dores distais à laringe. Foram consideradas como dores proximais: dor na ATM/Mandíbulla, dor na língua, dor na garganta, dor na nuca, dor nos ombros, dor no pescoço e dor ao falar. Já as dores distais foram: dor de cabeça, dor nas costas/coluna, dor no peito, dor nos braços, dor nas mãos e dor nos ouvidos.

A frequência das dores também foi agrupada para melhor análise dos dados. A opção "nunca" foi considerada como "ausência de dor corporal". As opções "às vezes", "muitas vezes", "quase sempre" e "sempre" foram consideradas como "presença de dor corporal".

Para a análise estatística foi utilizado o programa SPSS (Statistical Package for Social Sciences), versão 19.0. O nível de significância adotado para todos os resultados foi de $5 \%$, $\mathrm{p} \leq 0,05$. Para comparar a autoavaliação vocal, as variáveis de relato de dor e a relação de dores com os dados referentes à voz foi aplicado o teste da Razão de Verossimilhança, ajustado pela Correção de Bonferroni. O Teste Exato de Fisher ajustado pela Correção de Bonferroni foi utilizado para tentarmos identificar se os grupos diferenciavam-se entre si em cada variável do tipo de dor. Foi realizado cálculo do odds-ratio cujo valor deve ser efetivamente maior do que um ou efetivamente menor do que um. Se o intervalo formado pelos limites inferior e superior não contiver o valor um, então, o valor de odds-ratio pode ser considerado efetivo, indicando diferença de risco por pertencer a um ou outro grupo. Também foi aplicado o Teste de Mann-Whitney, ajustado pela Correção de Bonferroni para verificar se os grupos diferenciavam-se em relação ao grupo de dores totais, proximais e distais.

\section{RESULTADOS}

A autoavaliação vocal foi semelhante entre os grupos $(\mathrm{p}=0,509)$ e mostrou que a maior parte dos sujeitos da população geral e dos coristas, considera a voz boa ( $48 \%$ população geral e 54\% dos coristas). A população geral mencionou, ainda, as opções razoável $(25,3 \%)$, ótima (24\%), ruim (2\%) e péssima $(0,7 \%)$. Para os coristas as classificações ótima e razoável tiveram a mesma ocorrência (20\% cada), seguida de ruim $(6 \%)$. Nenhum corista classificou a própria voz como péssima.

Os valores referentes à presença ou ausência dos 13 tipos de dores corporais pesquisados revelam que a frequência de todos eles foi maior na população geral (Tabela 1). Observamos que não há qualquer relação entre as dores corporais e outras variáveis como problema de voz, cancelamento de apresentação, consulta com otorrinolaringologista e consulta com fonoaudiólogo no grupo de coristas eruditos. Porém, as consultas com fonoaudiólogo foram associadas à dor na ATM e dor ao falar.

A média de dores foi maior para a população geral. A ocorrência de dores proximais não diferenciou os grupos. Porém quando comparamos o número total de dores ou apenas as dores distais, observamos maior ocorrência entre os sujeitos da população geral (Tabela 2).
O cálculo do odds-ratio mostrou que ser corista erudito pode ser considerado fator de proteção para a ocorrência de dores na cabeça, nas costas, no pescoço, nos braços, nas mãos e nos ouvidos (Tabela 3).

\section{DISCUSSÃO}

Segundo a Sociedade Brasileira de Estudos da Dor (SBED) ${ }^{(9)}$ fatores biológicos, sociais e psicológicos influenciam na sensação de dor e, além disso, cada individuo classifica e interpreta suas dores de acordo com experiências anteriores. Pesquisas demonstram que a percepção da dor está intimamente relacionada ao estado emocional das pessoas ${ }^{(10)}$.

Em nossa pesquisa, observamos que, em 12 das 13 dores pesquisadas, coristas eruditos relataram menos dores corporais do que a população geral. A única exceção foi a o item dor na garganta, que será explorado mais adiante (Tabela 1). Como cantores eruditos possuem uma rotina de exercícios de fortalecimento e relaxamento da musculatura envolvida na produção $\operatorname{vocal}^{(7)}$, é possível que este treinamento contribua para uma menor ocorrência das dores por favorecer o desenvolvimento de maior resistência muscular. Estes resultados podem ser atribuídos não somente aos benefícios fisiológicos proporcionados pela prática do canto, mas também aos benefícios psicológicos, já que a prática do coral é frequentemente encarada como uma atividade prazerosa ${ }^{(2)}$.

Quando dividimos as dores estudadas em distais e proximais observamos que não houve diferença entre os grupos para ocorrência de dores proximais. Já a ocorrência de dores distais foi superior entre os sujeitos da população geral (Tabela 2). Ao compararmos as chances de um corista erudito e de um sujeito da população geral apresentar dores corporais, observamos que o risco entre os sujeitos da população normal é maior. Isso indica que praticar canto erudito pode ser um fator de proteção, visto que todos os participantes do coral exercem alguma atividade profissional paralela não necessariamente ligada ao canto. Este fator de proteção foi mais expressivo para as dores na cabeça, nas costas, no pescoço, nos braços, nas mãos e nos ouvidos (Tabela 3).

Segundo a literatura, os principais problemas encontrados entre os cantores estão relacionados à higiene vocal inadequada, na qual podemos incluir o uso incorreto de técnicas respiratórias, grande tensão musculoesquelética e desequilíbrio ressonantal ${ }^{(11)}$. Pesquisas demonstram que cantores treinados possuem um controle da dinâmica respiratória, laríngea e articulatória mais desenvolvido ${ }^{(12)}$ e que, quanto mais treinada a voz, melhor a simetria de vibração das pregas vocais e a linearidade do sistema muscular ${ }^{(13)}$.

No canto, os sintomas de dor são geralmente secundários à tensão muscular realizada para compensar uma técnica vocal inadequada $^{(4,14)}$. Talvez tenhamos encontrado baixos relatos de dor em nossos coristas devido ao treinamento intensivo e excelente técnica vocal exigida a todos os participantes deste coral. Acreditamos que não só o treino como também um melhor conhecimento, percepção e controle das estruturas envolvidas na produção da voz estejam diretamente relacionados com o resultado desta pesquisa. 
Tabela 1. Relação entre a ocorrência de dores com as variáveis referentes a problemas de voz durante o exercício profissional

\begin{tabular}{|c|c|c|c|c|c|c|c|c|c|c|}
\hline & \multicolumn{5}{|c|}{ Coristas eruditos } & \multicolumn{5}{|c|}{ População geral } \\
\hline & \multicolumn{2}{|c|}{ Sim } & \multicolumn{2}{|c|}{ Não } & \multirow{2}{*}{ Valor de $p$} & \multicolumn{2}{|c|}{ Sim } & \multicolumn{2}{|c|}{ Não } & \multirow{2}{*}{ Valor de $p$} \\
\hline & $\mathrm{n}$ & $\%$ & $\mathrm{n}$ & $\%$ & & $\mathrm{n}$ & $\%$ & $\mathrm{n}$ & $\%$ & \\
\hline Já experienciou problema de voz & 19 & 38 & 31 & 62 & & 43 & 28,6 & 107 & 71,3 & \\
\hline Dor na cabeça & 9 & 18 & 41 & 82 & $>0,999$ & 70 & 46,7 & 80 & 53,3 & 0,103 \\
\hline Dor na ATM & 7 & 14 & 43 & 86 & $>0,999$ & 39 & 26 & 111 & 74 & $0,001^{*}$ \\
\hline Dor na língua & 2 & 4 & 48 & 96 & $>0,999$ & 12 & 8 & 138 & 92 & $>0,999$ \\
\hline Dor na garganta & 28 & 56 & 22 & 44 & $>0,999$ & 79 & 52 & 71 & 47,3 & $0,011^{*}$ \\
\hline Dor na nuca & 12 & 24 & 38 & 76 & $>0,999$ & 57 & 38 & 93 & 62 & $<0,001^{*}$ \\
\hline Dor nos ombros & 15 & 30 & 35 & 70 & $>0,999$ & 68 & 45,3 & 82 & 54,7 & $<0,001^{*}$ \\
\hline Dor nas costas & 19 & 38 & 31 & 62 & $>0,999$ & 87 & 58 & 63 & 42 & $0,011^{*}$ \\
\hline Dor no pescoço & 13 & 26 & 37 & 74 & $>0,999$ & 74 & 49,3 & 76 & 50,7 & $0,019^{*}$ \\
\hline Dor no peito & 0 & 0 & 50 & 100 & $>0,999$ & 37 & 24,7 & 113 & 75,3 & 0,093 \\
\hline Dor nos braços & 4 & 8 & 46 & 92 & $>0,999$ & 44 & 29,3 & 106 & 70,7 & $0,005^{*}$ \\
\hline Dor nas mãos & 3 & 6 & 47 & 94 & $>0,999$ & 38 & 25,3 & 112 & 74,7 & 0,100 \\
\hline Dor nos ouvidos & 1 & 2 & 49 & 98 & $>0,999$ & 37 & 24,7 & 113 & 75,3 & $<0,001^{*}$ \\
\hline Dor ao falar & 10 & 20 & 40 & 80 & $>0,999$ & 19 & 12,7 & 131 & 87,3 & $<0,001^{*}$ \\
\hline $\begin{array}{l}\text { Cancelamento apresentação/ } \\
\text { trabalho por problema de voz }\end{array}$ & 8 & 16 & 42 & 84 & & 8 & 5,3 & 142 & 94,7 & \\
\hline Dor na cabeça & 9 & 18 & 41 & 82 & $>0,999$ & 70 & 46,7 & 80 & 53,3 & 0,147 \\
\hline Dor na ATM & 7 & 14 & 43 & 86 & $>0,999$ & 39 & 26 & 111 & 74 & 0,206 \\
\hline Dor na língua & 2 & 4 & 48 & 96 & $>0,999$ & 12 & 8 & 138 & 92 & $>0,999$ \\
\hline Dor na garganta & 28 & 56 & 22 & 44 & $>0,999$ & 79 & 52 & 71 & 47,3 & 0,066 \\
\hline Dor na nuca & 12 & 24 & 38 & 76 & $>0,999$ & 57 & 38 & 93 & 62 & 0,479 \\
\hline Dor nos ombros & 15 & 30 & 35 & 70 & $>0,999$ & 68 & 45,3 & 82 & 54,7 & 0,469 \\
\hline Dor nas costas & 19 & 38 & 31 & 62 & $>0,999$ & 87 & 58 & 63 & 42 & 0,469 \\
\hline Dor no pescoço & 13 & 26 & 37 & 74 & $>0,999$ & 74 & 49,3 & 76 & 50,7 & 0,491 \\
\hline Dor no peito & 0 & 0 & 50 & 100 & $>0,999$ & 37 & 24,7 & 113 & 75,3 & 0,680 \\
\hline Dor nos braços & 4 & 8 & 46 & 92 & $>0,999$ & 44 & 29,3 & 106 & 70,7 & $0,048^{*}$ \\
\hline Dor nas mãos & 3 & 6 & 47 & 94 & $>0,999$ & 38 & 25,3 & 112 & 74,7 & $>0,999$ \\
\hline Dor nos ouvidos & 1 & 2 & 49 & 98 & $>0,999$ & 37 & 24,7 & 113 & 75,3 & 0,408 \\
\hline Dor ao falar & 10 & 20 & 40 & 80 & $>0,999$ & 19 & 12,7 & 131 & 87,3 & $0,009^{*}$ \\
\hline Consulta ORL por problema de voz & 16 & 32 & 34 & 68 & & 27 & 18 & 123 & 82 & \\
\hline Dor de cabeça & 9 & 18 & 41 & 82 & $>0,999$ & 70 & 46,7 & 80 & 53,3 & $>0,999$ \\
\hline Dor ATM & 7 & 14 & 43 & 86 & $>0,999$ & 39 & 26 & 111 & 74 & $0,007^{*}$ \\
\hline Dor na língua & 2 & 4 & 48 & 96 & $>0,999$ & 12 & 8 & 138 & 92 & $>0,999$ \\
\hline Dor na garganta & 28 & 56 & 22 & 44 & $>0,999$ & 79 & 52 & 71 & 47,3 & 0,290 \\
\hline Dor na nuca & 12 & 24 & 38 & 76 & $>0,999$ & 57 & 38 & 93 & 62 & $0,049^{*}$ \\
\hline Dor nos ombros & 15 & 30 & 35 & 70 & $>0,999$ & 68 & 45,3 & 82 & 54,7 & 0,136 \\
\hline Dor nas costas & 19 & 38 & 31 & 62 & $>0,999$ & 87 & 58 & 63 & 42 & 0,197 \\
\hline Dor no pescoço & 13 & 26 & 37 & 74 & $>0,999$ & 74 & 49,3 & 76 & 50,7 & 0,292 \\
\hline Dor no peito & 0 & 0 & 50 & 100 & $>0,999$ & 37 & 24,7 & 113 & 75,3 & 0,323 \\
\hline Dor nos braços & 4 & 8 & 46 & 92 & $>0,999$ & 44 & 29,3 & 106 & 70,7 & $0,034^{*}$ \\
\hline Dor nas mãos & 3 & 6 & 47 & 94 & $>0,999$ & 38 & 25,3 & 112 & 74,7 & 0,144 \\
\hline Dor nos ouvidos & 1 & 2 & 49 & 98 & $>0,999$ & 37 & 24,7 & 113 & 75,3 & $0,047^{\star}$ \\
\hline Dor ao falar & 10 & 20 & 40 & 80 & $>0,999$ & 19 & 12,7 & 131 & 87,3 & $0,001^{*}$ \\
\hline Consulta Fono por problema de voz & 12 & 24 & 38 & 76 & & 28 & 18,6 & 122 & 81,4 & \\
\hline Dor na cabeça & 9 & 18 & 41 & 82 & 0,425 & 70 & 46,7 & 80 & 53,3 & 0,294 \\
\hline Dor na ATM & 7 & 14 & 43 & 86 & $0,048^{*}$ & 39 & 26 & 111 & 74 & $0,003^{*}$ \\
\hline Dor na língua & 2 & 4 & 48 & 96 & $>0,999$ & 12 & 8 & 138 & 92 & 0,466 \\
\hline Dor na garganta & 28 & 56 & 22 & 44 & $>0,999$ & 79 & 52 & 71 & 47,3 & 0,210 \\
\hline Dor na nuca & 12 & 24 & 38 & 76 & $>0,999$ & 57 & 38 & 93 & 62 & $0,009^{*}$ \\
\hline Dor nos ombros & 15 & 30 & 35 & 70 & $>0,999$ & 68 & 45,3 & 82 & 54,7 & 0,092 \\
\hline Dor nas costas & 19 & 38 & 31 & 62 & 0,171 & 87 & 58 & 63 & 42 & 0,056 \\
\hline Dor no pescoço & 13 & 26 & 37 & 74 & 0,707 & 74 & 49,3 & 76 & 50,7 & 0,095 \\
\hline Dor no peito & 0 & 0 & 50 & 100 & $>0,999$ & 37 & 24,7 & 113 & 75,3 & 0,149 \\
\hline Dor nos braços & 4 & 8 & 46 & 92 & $>0,999$ & 44 & 29,3 & 106 & 70,7 & $0,038^{*}$ \\
\hline Dor nas mãos & 3 & 6 & 47 & 94 & $>0,999$ & 38 & 25,3 & 112 & 74,7 & 0,227 \\
\hline Dor nos ouvidos & 1 & 2 & 49 & 98 & 0,240 & 37 & 24,7 & 113 & 75,3 & $0,026^{*}$ \\
\hline Dor ao falar & 10 & 20 & 40 & 80 & $0,046^{*}$ & 19 & 12,7 & 131 & 87,3 & $0,002^{*}$ \\
\hline
\end{tabular}

*Valores significativos $(p \leq 0,05)$ - Teste Exato de Fisher

Legenda: $\mathrm{ATM}$ = articulação temporomandibular; ORL = otorrinolaringologista 
Tabela 2. Comparação da ocorrência de dores totais, proximais e distais à laringe entre coristas e a população geral

\begin{tabular}{|c|c|c|c|c|c|c|c|}
\hline \multirow{2}{*}{ Quantidade de dores } & \multicolumn{3}{|c|}{ Coristas } & \multicolumn{3}{|c|}{ População geral } & \multirow{2}{*}{ Valor de $\mathrm{p}$} \\
\hline & $\mathrm{n}$ & Média & Desvio-padrão & $\mathrm{n}$ & Média & Desvio-padrão & \\
\hline Totais & 50 & 2,46 & 2,04 & 150 & 4,41 & 3,41 & $<0,001^{*}$ \\
\hline Proximais à laringe & 50 & 1,74 & 1,48 & 150 & 2,32 & 1,95 & 0,108 \\
\hline Distais à laringe & 50 & 0,72 & 0,88 & 150 & 2,09 & 1,75 & $<0,001^{*}$ \\
\hline
\end{tabular}

*Valores significativos $(p \leq 0,05)$ - Teste Mann-Whitney, ajustado pela correção de Bonferroni

Tabela 3. Comparação das chances de ocorrência de cada tipo de dor entre a população geral e os cantores eruditos

\begin{tabular}{|c|c|c|c|c|c|c|c|c|c|c|c|c|c|c|}
\hline Grupos & Estatística & $\begin{array}{c}\text { Dor } \\
\text { cabeça }\end{array}$ & $\begin{array}{l}\text { Dor } \\
\text { ATM }\end{array}$ & $\begin{array}{c}\text { Dor } \\
\text { língua }\end{array}$ & $\begin{array}{c}\text { Dor } \\
\text { garganta }\end{array}$ & $\begin{array}{c}\text { Dor } \\
\text { nuca }\end{array}$ & $\begin{array}{c}\text { Dor } \\
\text { ombros }\end{array}$ & $\begin{array}{c}\text { Dor } \\
\text { costas }\end{array}$ & $\begin{array}{c}\text { Dor } \\
\text { pescoço }\end{array}$ & $\begin{array}{l}\text { Dor } \\
\text { peito }\end{array}$ & $\begin{array}{c}\text { Dor } \\
\text { braços }\end{array}$ & $\begin{array}{l}\text { Dor } \\
\text { mão }\end{array}$ & $\begin{array}{c}\text { Dor } \\
\text { ouvidos }\end{array}$ & $\begin{array}{l}\text { Dor } \\
\text { falar }\end{array}$ \\
\hline População & Odds-ratio & $3,98^{*}$ & 2,15 & 2,08 & 0,87 & 1,94 & 1,93 & $2,25^{\star}$ & $2,771^{*}$ & - & $4,77^{*}$ & $5,31^{*}$ & $16,04^{*}$ & 0,58 \\
\hline $\begin{array}{l}\text { Geral } \\
\text { versus }\end{array}$ & Limite Inferior & 1,81 & 0,89 & 0,45 & 0,45 & 0,93 & 0,97 & 1,16 & 1,365 & - & 1,60 & 1,56 & 2,14 & 0,25 \\
\hline $\begin{array}{l}\text { Cantor } \\
\text { Erudito }\end{array}$ & Limite superior & 8,77 & 5,19 & 9,66 & 1,66 & 4,01 & 3,83 & 4,34 & 5,627 & - & 14,06 & 18,07 & 120,2 & 1,34 \\
\hline
\end{tabular}

*Valores significativos $(p \leq 0,05)$ - Odds-ratio

Legenda: ATM = articulação temporomandibular

Cantores geralmente possuem maior sensibilidade e percepção a respeito de tudo que está relacionado à voz e, às vezes, associam dores corporais a mudanças na qualidade vocal ${ }^{(3,4)}$. Esta relação não foi observada neste estudo. Os coristas eruditos não apresentaram relação entre dor e a ocorrência de problemas vocais ou com a necessidade de cancelar apresentações (Tabela 1).

A tensão mandibular tornou-se uma queixa muito frequente entre os cantores ${ }^{(15)} \mathrm{e}$, embora poucos coristas eruditos de nosso estudo tenham-na relatado, a dor na ATM e dor para falar foram relacionadas à procura por consulta com fonoaudiólogo (Tabela 1). Esta relação pode indicar que o fonoaudiólogo é reconhecido pelos coristas como um profissional que trata de dores na região facial e também da $\mathrm{voz}^{(16)}$.

Apesar do relato geral de dor entre os coristas ter sido menor do que na população geral (Tabelas 1 e 2), algumas considerações sobre as dores mais relatadas por esse grupo são importantes. A dor de garganta foi a mais relatada, o que pode ser explicado pelo alto nível de exigência vocal deste grupo de profissionais, já que o pertencem à elite vocal dos profissionais da voz. Sendo assim, sabe-se que qualquer alteração vocal ou laríngea (que pode vir acompanhada de dor) representa um grande problema para esta população ${ }^{(17)}$.

A dor nas costas foi a segunda mais referida entre os coristas eruditos. Cantores geralmente ficam sentados enquanto treinam ou ensaiam. Muitos estudos apontam que o alinhamento postural pode interferir diretamente na atividade muscular respiratória e consequentemente na técnica vocal, podendo causar dor ${ }^{(18,19)}$. Além disso, os ensaios de coral geralmente são longos, o que pode intensificar os danos causados por um alinhamento postural inadequado.

O terceiro tipo de dor mais relatado pelos coristas foi a dor nos ombros. Embora especialistas em voz aconselhem que não haja movimentação do pescoço e dos ombros durante a fonação, é possível que a musculatura dessas estruturas desenvolva atividade excessiva durante o processo fonatório ${ }^{(20)}$. Tensão muscular excessiva é um erro comum no canto ${ }^{(2)}$, além de ser a principal responsável pela dor referida por pacientes de voz, levando a uma sequência de eventos que desequilibram o sistema muscular ${ }^{(2,4)}$.

O grupo de coristas eruditos relatou menos dor no pescoço que a população geral (Tabela 1), e esta diferença aconteceu provavelmente devido ao bom treinamento vocal deste grupo. O efeito do treino vocal parece ter grande influência no desenvolvimento e aprimoramento da produção e percepção da voz como um conjunto de ações e reações ${ }^{(7,21)} \mathrm{e}$, além disso, corresponde a um método de prevenção primária contra distúrbios de voz ${ }^{(22)}$.

Com esse estudo podemos inferir que a prática do canto e o uso vocal consciente contribuem para menores índices de dores corporais, provavelmente por conta das técnicas de produção de voz e de respiração bem desenvolvidas e também pela sensação de bem-estar proporcionada por esta atividade. Em novos estudos, serão feitas comparações entre os níveis de dores corporais em coristas eruditos e outros profissionais da voz, a fim de verificar se o bom treino vocal tem relação com o índice de dores corporais e consequentemente com a qualidade de vida dos indivíduos que utilizam a voz como ferramenta de trabalho.

\section{CONCLUSÃO}

Coristas eruditos apresentaram baixos índices dores corporais em relação à população geral. Esse baixo relato de dores corporais provavelmente se deve a uma melhor resistência muscular adquirida ao longo dos constantes treinamentos e práticas, indispensáveis aos cantores eruditos.

Diferentemente da população geral, os coristas eruditos não têm dores associadas a problemas vocais e absenteísmo. O uso vocal de forma consciente aliado ao treino pode ser um fator de proteção contra o aparecimento de distúrbios vocais e dores em geral.

*TV colaborou com a coleta e análise de dados e elaboração do manuscrito; ACG colaborou com a revisão da literatura, coleta e análise de dados; MB colaborou com o delineamento da pesquisa, orientação do estudo e correção do texto. 


\section{REFERÊNCIAS}

1. International Association for the Study of Pain (IASP). 2008. [cited 2008 Jan 28]. Available from: http://www.iasp-pain.org

2. Arboleda BMW, Frederick AL. Consideration for maintenance of postural alignment for voice production. J Voice. 2008;22(1):90-9.

3. Smith BJ, Sataloff RT. Choral pedagogy. San Diego: Singular Publishing Group; 2000.

4. Sataloff RT, Divi V, Heman-Ackah YD, Hawkshaw MJ. Medical history in voice professionals. Otolaryngol Clin North Am. 2007;40(5):931-51.

5. Rubin JS, Blake E, Mathieson L. Musculoskeletal patterns in patients with voice disorders. J Voice. 2007;21(4):477-84.

6. Demmink-Geertman L, Dejonckere PH. Neurovegetative symptoms and complaints before and after voice therapy for nonorganic habitual dysphonia. J Voice. 2008;22(3):315-25.

7. Braun-Janzen C, Zeine L. Singers' interest and knowledge levels of vocal function and dysfunction: survey findings. J Voice. 2009;23(4):470-83.

8. Scheffel L. Presença de dores corporais relacionadas ao uso da vozemprofessores do ensinofundamental da Rede Escolar Municipal da Cidade de Novo Hamburgo-RS [thesis]. São Paulo: Center For Vocal Studies; 2006.

9. Sociedade Brasileira de Estudos da Dor (SBED). 2011. [cited 2011 Nov 02]. Available from: http://www.dor.org.br

10. Kut E, Schaffner N, Wittwer A, Candia V, Brockmann M, Storck C, et al. Changes in self-perceived role identity modulate pain perception. Pain. 2007;131(1-2):191-201.

11. Wilner LK, Sataloff RT. Speech-language pathology and the professional voice. Ear Nose Throat J. 1987;66(8):313-7.
12. Teachey JC, Kahane JC, Beckford NS. Vocal mechanics in untrained professional singers. J Voice. 1991;5(1):51-6.

13. Pontes P, Brasolotto A, Behlau M. Glottic characteristics and voice complaint in elderly. J Voice. 2005;19(1):84-94.

14. Spiegel JR, Sataloff RT, Cohn JR, Hawkshaw M, Epstein J. Respiratory function in singers: medical assessment, diagnoses, and treatments. J Voice. 1988;2(1):40-50.

15. Miller R. On the art of singing. Oxford University Press: New York; 1996.

16. Donnarumma, MDC, Muzilli CA, Ferreira C, Nemr K. Disfunções temporomandibulares: sinais, sintomas e abordagem multidisciplinar Rev CEFAC. 2010;12(5):788-94.

17. Koufman JA, Isaacson G. The spectrum of vocal dysfunction. Otolaryngol Clin North Am. 1991;24(5):985-8.

18. Staes FF, Jansen L, Vilette A, Coveliers Y, Daniels K, Decoster W. Physical therapy as a means to optimize posture and voice parameters in student classical singers: a case report. J Voice. 2011;25(3): 91-101.

19. Norris R. Seating problems of vocalists. In: Professional Voice: the science and art of clinical care. San Diego: Plural Publishing; 2005. p. 707-09.

20. Pettersen V, Westgaard RH. The activity patterns on neck muscles in professional classical singing. J Voice. 2005;19(2):238-51.

21. Mendes AP, Rothman HB, Sapienza C, Brown WS Jr. Effects of vocal training on the acoustic parameters of the singing voice. J Voice. 2003;17(4):529-43.

22. Borch DZ, Sundberg J. Some phonatory and resonatory characteristics of the rock, pop, soul and Swedish dance band styles of singing. J Voice. 2010;6(1):1-6.

Anexo 1. Protocolo de autoavaliação para coristas eruditos

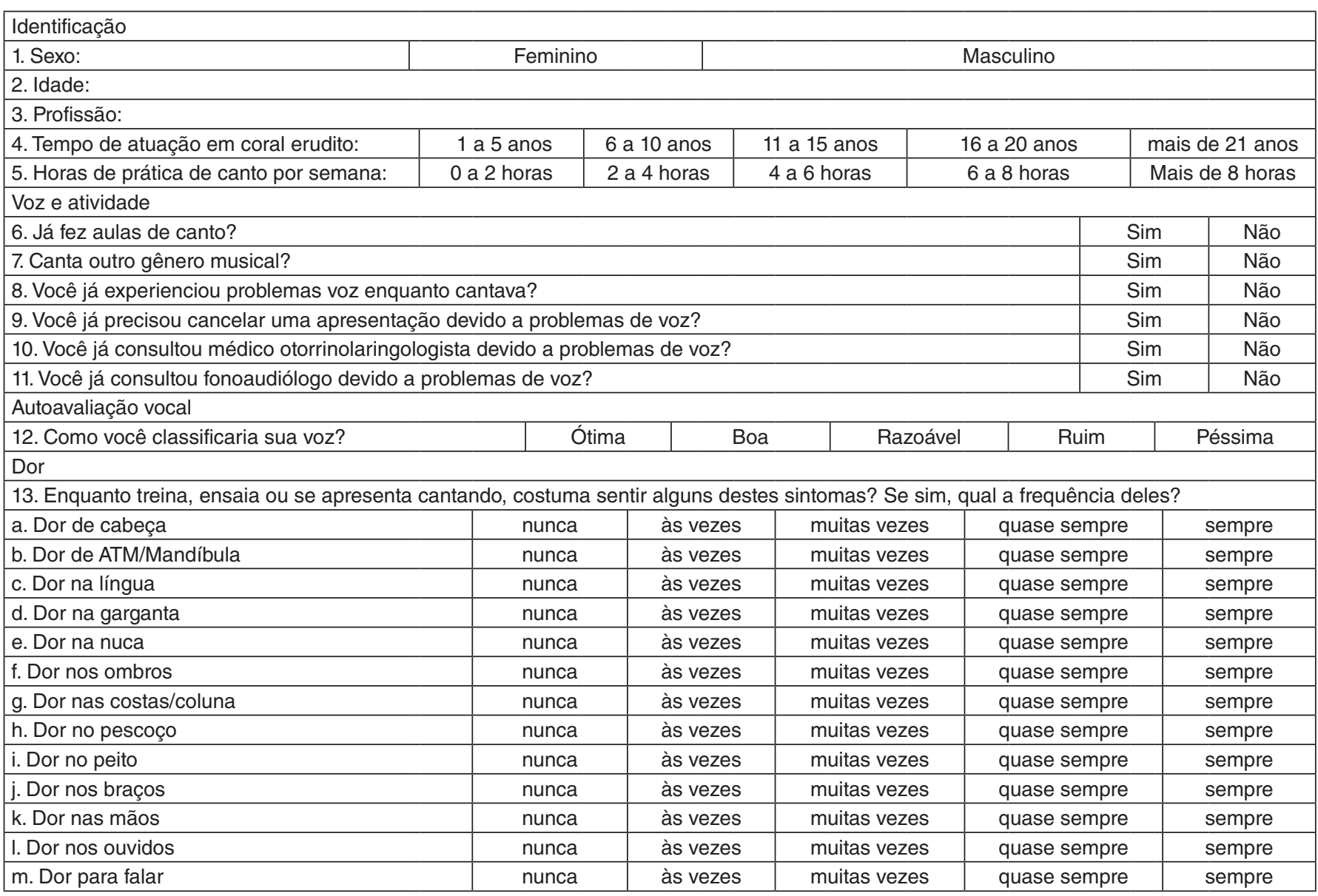

Legenda: ATM = articulação temporomandibular 
Anexo 2. Protocolo de autoavaliação para sujeitos da população geral

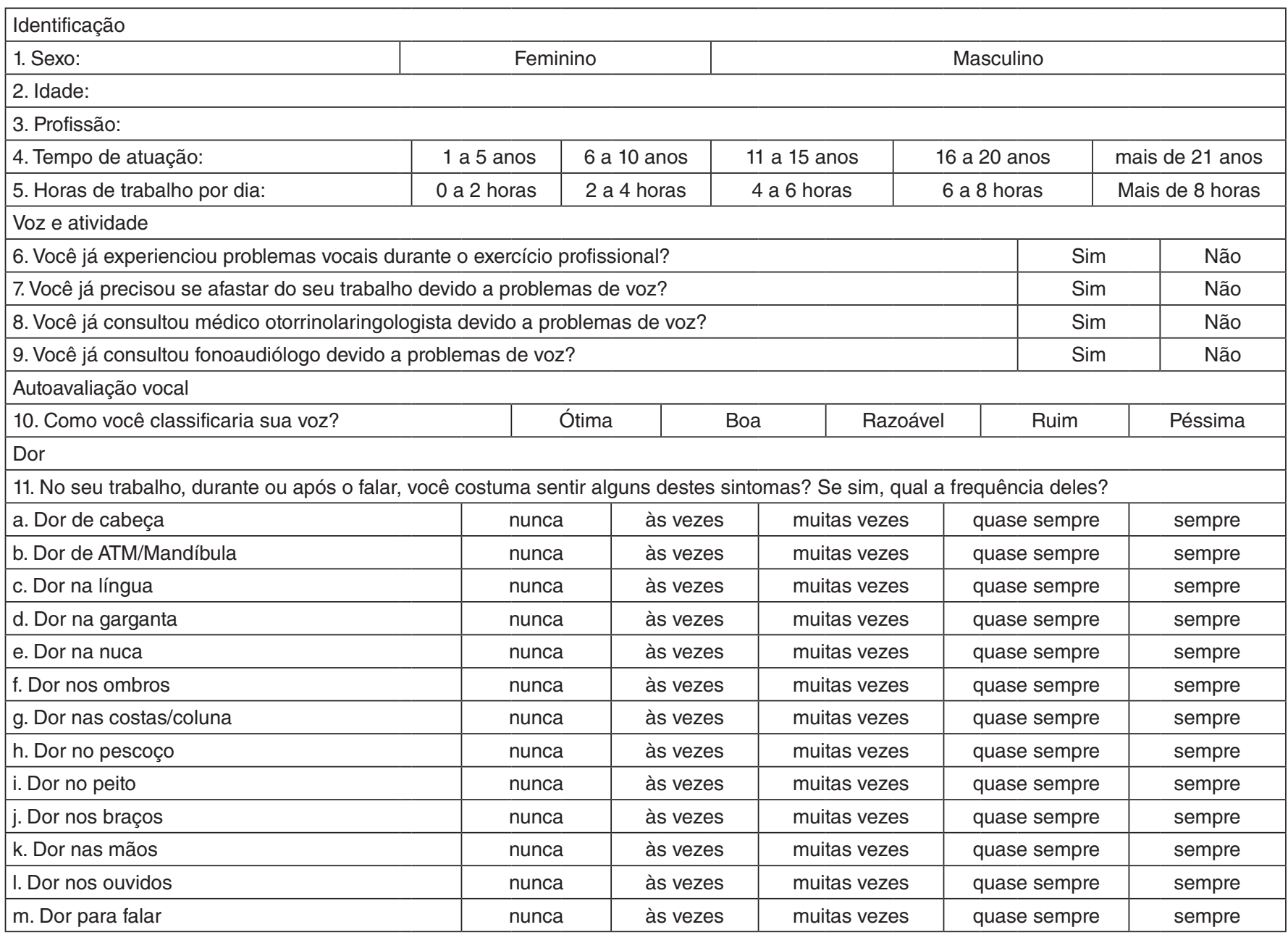

Legenda: ATM = articulação temporomandibular 